\title{
Signal Enhancements in Plasmon Assisted Fluorescence Ratiometric Imaging Optodes
}

\author{
Niklas Strömberg \\ SP Technical Research Institute of Sweden, Borås, Sweden \\ Email: niklas.stromberg@sp.se
}

Received November 16, 2012; revised December 17, 2012; accepted December 25, 2012

\begin{abstract}
The objective of this study was to benchmark the different signal enhancements found in a recently exploited fluorescence ratiometric optode design used for nano-molar imaging of ammonium and ammonia. The sensing scheme of these optodes are based on a mediated transfer of the analyte together with a fluorescent dye in a two-phase system consisting of a gold nanoparticle (GNP) doped ether (organic phase), emulsified in a hydrogel (hydrous phase). The coextraction of the ion dye pair causes changes in fluorescence in relation to the analyte concentration. Performances of optodes with and without GNPs using the gradually improved instrumentation and signal processing were evaluated and normalized to be comparable. Signal to noise was enhanced due to signal processing based on ensemble averaging $(1.7 \times-3.2 \times)$, CCD sensitivity $(2 \times)$, and plasmon assisted fluorescence $(10 \times-100 \times)$, which altogether with the ratiometric treatment of the fluorescence contributed to the great sensitivity for ammonium and ammonia. The study shows that GNP doped sensors are relatively more sensitive to matrix effects but if they are isolated by a protective layer they will dramatically increase in sensitivity. Proper isolation of the active chemical components from the matrix will make the sensor design one of the most powerful and versatile concepts for chemical imaging and single point detection in complex environments as the optodes likely can be constructed for most ions that have selective ionophores.
\end{abstract}

Keywords: Ions; Nanoparticles; Plasmonics; Plasmophore; Plasmons; Optode; Fluorosensor; Imaging; Ammonia; Ammonium

\section{Introduction}

The idea with this paper was to illustrate and discuss the remarkable sensitivity associated with a versatile (applicable to many ions) fluorescence ratiometric plasmon assisted imaging optode design. The optodes based on this design have not only been claimed to be the most sensitive sensor technique for noninvasive imaging of ammonia and ammonium, but also most likely the most sensitive technique for single point measurements of these species. Moreover, the versatile sensing scheme is transferable to other ions that have selective ionophores providing future expected great selectivities and sensitivities for a wide range of analytes.

The original physical sensor configuration, with an emulsion of an organic phase stabilized in a hydrogel, ionophore and a solvatochromic dye was presented already in 1999 by Krause et al. [1]. In the emulsion the negatively charged fluorescent dye (MC540) and a carrier molecule (in their case Valinomycin for potassium) coextract the ion from the hydrous phase into the organic phase that resulted in an increased fluorescence emission and redshift from $\sim 570 \mathrm{~nm}$ to $\sim 590 \mathrm{~nm}$ due to the solvatochromic dye. This concept makes it possible to detect more than 40 different ions in the same way (same excitation and emission) by just changing the ionophore or ionophore dye pair for negative ions, which also was done in several of the following publications [1-5]. When the sensor concept was transferred to ammonium the cross-sensitivity for potassium (main interfering ion) was found to be low and even better that reported for ion selective electrodes [5]. The technique was also claimed to be $\mathrm{pH}$ insensitive and virtually independent of ionic strength [1] of which the latter was found to be true up to $200 \mathrm{mM}$ [6]. However, even if the sensing concept was good and general, it was far from functioning for imaging and also questionable to function for single point measurements. The argument for this is that the fluorescent signal was not normalized and thus sensitive for changes in excitation light intensities, dye leakage and photo bleaching, making accurate detection of ions difficult in reality. A ratiometric concept was published in 2003 that not only compensated for excitation light changes but also made the sensor completely $\mathrm{pH}$ independent in the interval pH $5-7$ [6]. The normalization technique make use of the fluorescence signals from both the dye in hydrous and organic phase in a fluorescence 
ratio (e.g. $589 \mathrm{~nm} / 568 \mathrm{~nm}$ ), which caused a signal to noise $(\mathrm{S} / \mathrm{N})$ improvement of up to $800 \%$ when high levels of ammonium $(10 \mathrm{mM})$ initially was tested. Upon ammonium exposure the signals representing the two phases are anti-correlated due to the dye phase transfer that results in an amplified ratio (F589 nm $\uparrow / F 568$ $\mathrm{nm} \downarrow)$. The majority of the observed increase in $\mathrm{S} / \mathrm{N}$ at high ammonium levels was attributed to the decrease in hydrous fluorescence (568 $\mathrm{nm}$ in Figure 1), a signal that was not utilized in previous studies. However the result was just presented in a poster [7] and not further discussed in the associated paper [6], since also the noise also was enhanced due to the ratiometric procedure at the low ammonium levels $(0.1 \mathrm{mM})$, giving an unaltered $\mathrm{S} / \mathrm{N}$ compared to single emission detection. This overlooked feature of the optode technique was later on experienced as the performance of the optode gradually got better without any changes in the optode configuration. It was caused by improved signal processing and low noise camera systems that lowered the noise and thereby improved $\mathrm{S} / \mathrm{N}$. The technique was ready for imaging but the initial tests showed that the ratiometric approach did not completely (though most of the drift was removed) compensate for dye leakage and photo bleaching over the sensor film as well as drift over time. This was however solved by using time correlated pixel-by-pixel calibration [8], a technique where each pixel was compensated for drift after the measurements through post processing. Practically this was done by using two calibrations one before and one after experiment which were linked together by a time function. The time for the image capture of the experimental images was then used to calculate the response curve in each pixel that in turn was used to calibrate fluorescent images into concentration images [8]. Also the analytical performance such as sensitivity, limit of detection (LOD), and expected operational lifetime were calculated for each pixel and presented as statistical images [9]. After improvements of the infinity corrected imaging system as well as a high throughput macro imaging techniques were developed [10], the technique was ready for research applications such as studying ammonium uptake close to roots [11], and multiple fertilizer release studies (using a high throughput imaging system) in different soils [12] over long time periods up to 11 days.

\section{Plasmon Assisted Fluorescence Ratiometric Imaging}

The last decade, lots of attention has been drawn to metal enhanced fluorescence techniques. The idea is to make use of metal particles that further reduce the noise and enhance the fluorescence intensities through so called plasmophore emissions [13]. The technique is applicable
Low Ammonium

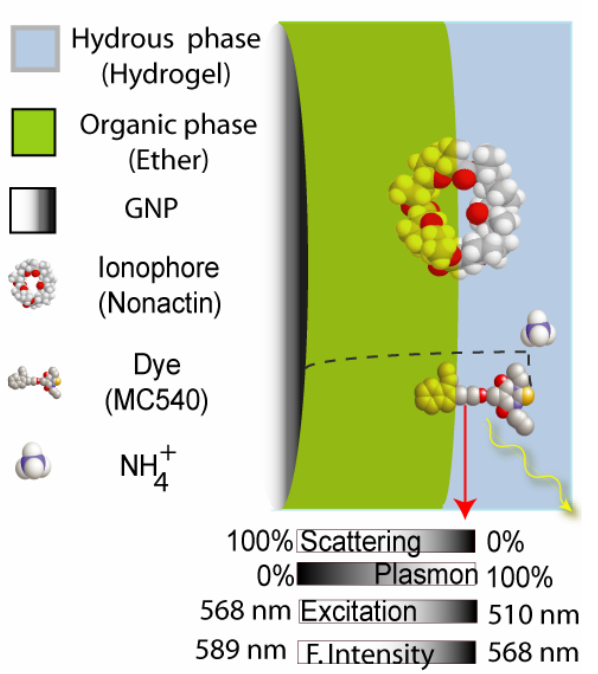

Figure 1. Principle of operation for the plasmon assisted optode at low ammonium concentration, with relative importance of the enhancements effects (black = low; white = high). Coupled plasmon assisted fluorescence (dashed line) GNP ex. = dye ex. @ $510 \mathrm{~nm}$; dye-GNP distance $\sim 20 \mathrm{~nm}$. Arrow (red) indicates the level of the enhancement effects.

if metal particles and fluorescence molecules are in a close vicinity of each other. Nanometer movements of dye molecules towards the metal surface are utilized to give metal enhanced fluorescence signals (plasmophores) as well as reduced chemical noise. As the sensing mechanism for the ionophore optode are based on nanometer movements of a dye molecule in an oil/water interphase, the plasmon assisted fluorescence technique would theoretically be successfully implemented in the sensor design. Especially when considering that the ratiometric procedure caused an $8 \times$ signal improvement at high ammonium concentrations could become a reality also for low concentrations by just reducing chemical noise. The physical sensor configuration was further improved in 2009 by incorporation of $10 \mathrm{~nm}$ thiol dervatized gold nanoparticles that were extracted into the organic phase of the sensor (see Figures $\mathbf{1}$ and $\mathbf{2}$ for principles of operation) [14]. At low ammonium levels the relatively lower absorbance (excitation in Figure 1), and fluorescence in the hydrous phase (hydrogel) are assisted by plasmons, as the distance $(10-20 \mathrm{~nm})$ match the far field emission region as well as the GNP/dye excitation wavelengths overlap. At high ammonium concentrations the plasmons are decoupled due to solvatochroism i.e. GNP/dye excitation do not overlap and fluorescence are increased due to the solvent shift and increased scattering $\left(\propto(\text { dye-GNP distance })^{2}\right)$. The initial tests showed a signal to noise $(\mathrm{S} / \mathrm{N})$ improvement surpassing three orders of magnitude ( $\sim 5000$ times) [14] compared to the initial ratiometric sensor [6] resulting in a LOD of $1.7 \mathrm{nM}$ for 
High Ammonium

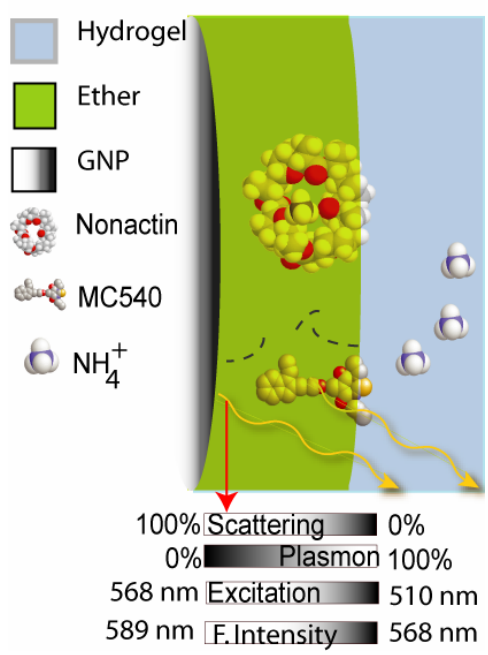

Figure 2. Principle of operation for the plasmon assisted optode at high ammonium concentration, with relative importance of the enhancements effects (black = low; white $=$ high). Decoupled plasmon assisted fluorescence (disrupted dashed line) due to solvatochroism. GNP ex $=510 \mathrm{~nm}$; dye ex $=568 \mathrm{~nm}$; dye-GNP distance $\sim 5 \mathrm{~nm}$. Arrow (red) indicates the level of the enhancement effects.

ammonium. The leverage in the normalized signal is likely powered by an emission increase due to GNP scattering in the organic phase as well as a reduction in noise in the hydrous phase together with increased hydrous plasmon assisted fluorescence which when utilized in the overall fluorescence ratio $(589 \mathrm{~nm} / 568 \mathrm{~nm})$ increase the $\mathrm{S} / \mathrm{N}$ dramatically. The concept was adapted for imaging using ratiometry and drift compensation in each pixel, with following remarkable sensitivities. In 2011 a sensor for detecting ammonia in tissues was presented
[15] along with an improved calibration function (further described in [16]) that resulted in a $2 \mathrm{nM}$ detection limit for ammonia at the pixel level in images.

\section{Experiment}

The previous work was compiled and each change or improvement of the technology was tracked and normalized in order to be comparable and thereafter benchmarked.

\section{Results and Discussion}

\subsection{Evaluation of Signal Enhancements}

In order to compare the sensitivities for the two different CCDs that used 3 and 10 images in the image average, the CCD system using 10 images was normalized to 3 images by making use of the principles for ensemble averaging [17] i.e. $\mathrm{S} / \mathrm{N} \propto(\mathrm{n} \text { images })^{0.5}$ (Table 1). Thereby, facilitating an estimate of the decrease in LOD attributed to the cooled CCD that turned out to be $\sim$ two times improved compared to the uncooled CCD using the same amount of image processing. The plasmon enhancement with high concentration $(\sim 30 \mu \mathrm{M})$ of metal particles improved the LOD 10 times compared to the LOD without GNPs. Even if this is far from the demonstrated more than 5000 times improvement in the spectrofluorometer, it is believed that the imaging optode was hampered by the matrix in this particular study of porcine tissue degradation. This suspicion was also supported when an ammonia sensor was tested in the same environment, where many interactions with the matrix were hindered by a gas permeable layer. The LOD improvement due to plasmon assisted fluorescence, as clearly can be judged by eye (Figure 3), was 100 times i.e. 1/100 of the LOD without GNPs.

Table 1. Performance of the optodes from 2001-2011 and corresponding factor improvement normalized to become comparable ${ }^{*} . \mathrm{S}=$ spectroflurometer; $\mathrm{CCD}=$ charged coupled device; $\mathrm{C}=$ cooled to $-30^{\circ} \mathrm{C} ; \mathrm{n}=$ number of images; $\mathrm{IA}=\mathrm{image}$ averaging; $M=$ median $3 \times 3$ filtration and $L O D=$ limit of detection.

\begin{tabular}{|c|c|c|c|c|c|c|c|}
\hline Measurement type & Specie & Plasmon & Signal processing & Reported LOD $(\mu \mathrm{M})$ & Instrument & Ref. & Factor \\
\hline Intensity & $\mathrm{NH}_{4}^{+}$ & - & - & $6-10$ & $\mathrm{~S}$ & {$[5]$} & \\
\hline Ratio & $\mathrm{NH}_{4}^{+}$ & - & - & $6-10$ & $\mathrm{~S}$ & {$[6]$} & $\sim 5000$ \\
\hline Ratio & $\mathrm{NH}_{4}^{+}$ & High & - & 0.0017 & $\mathrm{~S}$ & {$[14]$} & \\
\hline Ratio & $\mathrm{NH}_{4}^{+}$ & - & $\begin{array}{c}\mathrm{IA}, \mathrm{n}=10, \mathrm{M} \\
(\mathrm{IA}, \mathrm{n}=3), \mathrm{M}^{*}\end{array}$ & $\begin{array}{l}0.7-1.2 \\
(2.2)\end{array}$ & CCD & {$[8,9]$} & $\sim 2$ \\
\hline Ratio & $\mathrm{NH}_{4}^{+}$ & - & $\mathrm{IA}, \mathrm{n}=3, \mathrm{M}$ & 1 & $\mathrm{CCD}(\mathrm{C})$ & {$[18]$} & \\
\hline Ratio & $\mathrm{NH}_{4}^{+}$ & Low & $\mathrm{IA}, \mathrm{n}=3, \mathrm{M}$ & 0.3 & $\mathrm{CCD}(\mathrm{C})$ & {$[18]$} & $\sim 10$ \\
\hline Ratio & $\mathrm{NH}_{4}^{+}$ & High & $\mathrm{IA}, \mathrm{n}=3, \mathrm{M}$ & 0.1 & $\mathrm{CCD}(\mathrm{C})$ & {$[18]$} & \\
\hline Ratio & $\mathrm{NH}_{3}$ & - & $\mathrm{IA}, \mathrm{n}=3, \mathrm{M}$ & 0.2 & $\mathrm{CCD}(\mathrm{C})$ & {$[15]$} & $\sim 100$ \\
\hline Ratio & $\mathrm{NH}_{3}$ & High & $\mathrm{IA}, \mathrm{n}=3, \mathrm{M}$ & 0.002 & $\mathrm{CCD}(\mathrm{C})$ & {$[15]$} & \\
\hline
\end{tabular}




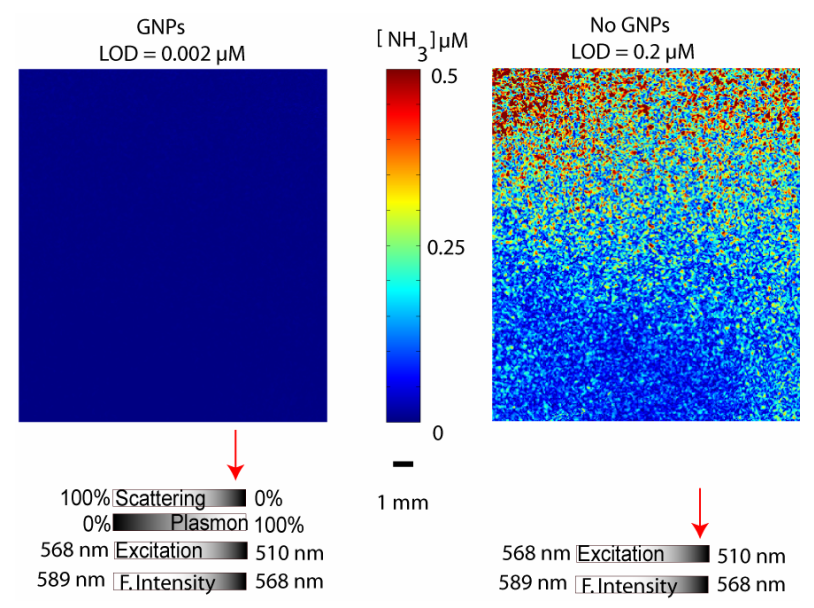

Figure 3. Comparison between ammonia optodes with and without GNPs with the associated enhancements effects for each sensor type. Arrows denote the likely relative contribution for each effect.

\subsection{Further Improvements of Plasmon Assisted Fluorescence}

Up to now, no optimization of the size, dervatization protocols and amount of GNPs have been done. Therefore further optimization of these parameters will likely result in improved imaging qualities such as improved LOD, analytical sensitivity and pixel to pixel noise. In addition, the complete technology has only been tested for ammonium and ammonia and there is still need to prove that this technology is transferable to the other ions with selective ionophores. If successfully implemented, this technology would significantly improve and simplify the development of artificial noses and tongues, as one detection system can be used to read out lots of different analytes when configured in arrays of optode patches. Moreover, the illumination system could be further improved to maintain stability over time. Initially xenon light sources were used for illumination but they were replaced by light emitting diodes (LEDs) due to their superior life time and relatively good intensity stability. However LEDs should ideally be temperature controlled in the same as the CCD chip or the intensity from the LEDs should be controlled by an intensity light feed-back system. So far no commercial LEDs are found (to the best of my knowledge) with this option and most LEDs just rely on a constant current operation, which insufficiently regulates the light intensities (especially during warm up). Hopefully scientific LED illumination will include this option in the near future. There shall also be mentioned that the CCD for ratiometric imaging optodes shall include a decent full well capacity $(>50,000$ electrons) a low root mean square (RMS) noise $(<15$ electrons) and a matched readout bit depth in order to have a sufficient dynamic range and enough readout levels. Prior to these publications presented here, tests were made with CCDs that did not fulfill these specifications which clearly limited the measurement performance.

For single point measurements there will even better possibilities for reduced detection limits and high sensitivities by making use of the imaging detector. Ensemble image and pixel averaging will make the noise reduction proportional to the square root of number of samplings or pixels [17]. If the commonly used pixel-by-pixel binning techniques are avoided and instead drift compensation in each pixel and thereafter pixel averaging over the entire CCD is used, the drift in the system will be removed and thus will not contribute to a biased signal. Such measurements strategies would theoretically reduce the noise completely e.g. with $10^{6}$ pixels averaging then the noise will be reduced by a factor of 1000 . This is especially an interesting approach for the development of highly sensitive artificial noses and tongues that do not need the image information and instead could be moved to each sensor spot through scanning.

\section{Conclusion}

It turns out that the presented imaging technology is enhanced approximately $1.7-3.2$ times using ensemble averaging ( 3 and 10 images), 2 times by using a cooled CCD compared to the un cooled CCD. For ammonium, LOD was improved 10 times due to the plasmophore enhancement. It seems like exclusion of the matrix effects further improved the LOD as the undoped ammonia detection (No GNPs) was improved 10 times compared to the undoped ammonium detection, relying on an extra gas barrier (No GNPs) othervise using the same sensor configuration, signal processing and detection system. When studying the plasmophore effect on ammonia detektion, the LOD was improved 100 times for ammonia with GNPs compared to ammonia detection without GNPs. However, in pure solutions with negligible matrix effects, a factor of 5000 can be attributed to the plasmophore effect alone. Thus, the compilation of these results strongly indicates that matrix effects are central for detection and regulates the sensitivity of the measurements for both sensors with and without GNPs. However, the plasmon assisted fluorescence are relatively more sensitive to matrix effects than ordinary fluorescence and thus isolation of the sensor from the matrix, with e.g. a molecular sieve or as in the ammonia example with a gas barrier, will likely pay off relatively more in terms of an improved LOD for plasmon based sensors. Still the S/N relation for the plasmon assisted fluorescent optode exceeds the original fluorescence ratiometric design with at least a factor of 10 . 


\section{Acknowledgements}

I greatly acknowledge my former supervisor Professor Stefan Hulth and my research colleague Aron Hakonen for their great contributions into this area.

\section{REFERENCES}

[1] C. Krause, T. Werner, C. Huber, O. S. Wolfbeis and M. J. P. Leiner, "pH-Insensitive Ion Selective Optode: A Coextraction-Based Sensor for Potassium Ions," Analytical Chemistry, Vol. 71, No. 8, 1999, pp. 1544-1548. doi:10.1021/ac981042x

[2] C. Huber, T. Werner, C. Krause, O. S. Wolfbeis and M. J. P. Leiner, "Overcoming the $\mathrm{pH}$ Dependency of Optical Sensors: A pH-Independent Chloride Sensor Based on Co-Extraction," Analytica Chimica Acta, Vol. 398, No. 2-3, 1999, pp. 137-143. doi:10.1016/S0003-2670(99)00414-6

[3] C. Krause, T. Werner, C. Huber and O. S. Wolfbeis, "Emulsion-Based Fluorosensors for Potassium Featuring Improved Stability and Signal Change," Analytical Chemistry, Vol. 71, No. 1999, pp. 5304-5308. doi:10.1021/ac9907383

[4] C. Krause, T. Werner and O. S. Wolfbeis, "Multilayer Potassium Sensor Based on Solid-State Coextraction," Analytical Sciences, Vol. 14, No. 1, 1998, pp. 163-167. doi:10.2116/analsci.14.163

[5] N. Stromberg and S. Hulth, "Ammonium Selective Fluorosensor Based on the Principles of Coextraction," Analytica Chimica Acta, Vol. 443, No. 2, 2001, pp. 215225. doi:10.1016/S0003-2670(01)01221-1

[6] N. Stromberg and S. Hulth, "A Fluorescence Ratiometric Detection Scheme for Ammonium Ions Based on the Solvent Sensitive Dye MC 540," Sensors and Actuators B: Chemical, Vol. 90, No. 1-3, 2003, pp. 308-318. doi:10.1016/S0925-4005(03)00053-4

[7] N. Strömberg and S. Hulth, "High-Precision Ratiometric Detection of $\mathrm{NH}^{4+}$ in Natural Waters," Europtode VI, Manchester, 2002.

[8] N. Stromberg and S. Hulth, "Assessing an Imaging Ammonium Sensor Using Time Correlated Pixel-by-Pixel calibration," Analytica Chimica Acta, Vol. 550, No. 1-2, 2005, pp. 61-68. doi:10.1016/j.aca.2005.06.074
[9] N. Stromberg and S. Hulth, "Time Correlated Pixelby-Pixel Calibration for Quantification and Signal Quality Control during Solute Imaging," Sensors and Actuators B: Chemical, Vol. 115, No. 1, 2006, pp. 263-269. doi:10.1016/j.snb.2005.09.012

[10] N. Stromberg, J. Engelbrektsson and S. Delin, "A High Throughput Optical System for Imaging Optodes," Sensors and Actuators B: Chemical, Vol. 140, No. 2, 2009, pp. 418-425. doi:10.1016/j.snb.2009.05.011

[11] N. Stromberg, "Determination of Ammonium Turnover and Flow Patterns Close to Roots Using Imaging Optodes," Environmental Science \& Technology, Vol. 42, No. 5, 2008, pp. 1630-1637. doi:10.1021/es071400q

[12] S. Delin and N. Stromberg, "Imaging-Optode Measurements of Ammonium Distribution in Soil after Different Manure Amendments," European Journal of Soil Science, Vol. 62, No. 2, 2011, pp. 295-304. doi:10.1111/j.1365-2389.2010.01326.x

[13] J. R. Lakowicz, K. Ray, M. Chowdhury, H. Szmacinski, Y. Fu, J. Zhang and K. Nowaczyk, "Plasmon-Controlled Fluorescence: A New Paradigm in Fluorescence Spectroscopy," Analyst, Vol. 133, No. 10, 2008, pp. 13081346. doi:10.1039/b802918k

[14] A. Hakonen, "Plasmon Enhancement and Surface Wave Quenching for Phase Ratiometry in Coextraction-Based Fluorosensors," Analytical Chemistry, Vol. 81, No. 11, 2009, pp. 4555-4559. doi:10.1021/ac8025866

[15] N. Strömberg and A. Hakonen, "Plasmophore Sensitized Imaging of Ammonia Release from Biological Tissues Using Optodes," Analytica Chimica Acta, Vol. 704, No. 1-2, 2011, pp. 139-145. doi:10.1016/j.aca.2011.08.019

[16] A. Hakonen and N. Stromberg, "Diffusion Consistent Calibrations for Improved Chemical Imaging Using Nanoparticle Enhanced Optical Sensors," Analyst, Vol. 137, No. 2, 2012, pp. 315-321. doi:10.1039/c1an15528h

[17] D. A. Skoog, F. J. Holler and T. A. Nieman, "Principles of Instrumental Analysis," In: F. Messina, Ed., Principles of Instrumental Analysis, Saunders Collage Publishing, Philadelphia, 1998, pp. 99-114.

[18] A. Hakonen and N. Stromberg, "Plasmonic Nanoparticle Interactions for High-Performance Imaging Fluorosensors," Chemical Communications, Vol. 47, No. 12, 2011, pp. 3433-3435. doi:10.1039/c0cc04972g 